\title{
Comment on: Traditional Chinese Eight Brocade Exercise Prescription for Ankylosing Spondylitis: A Quantitative Synthesis
}

\author{
Yu-Hui He \\ Department of Medicine, Peking University, Beijing, China
}

\section{Dear Editor,}

I came across the article accidentally entitled "Traditional Chinese Eight Brocade Exercise Prescription for Ankylosing Spondylitis: A Quantitative Synthesis," which was published online on May 28, 2020, in Complementary Medicine Research [1]. There are some viewpoints to be discussed.

This paper is a quantitative synthesis to assess the safety and effectiveness of traditional Chinese Eight Brocade exercise (also called BaDuan Jin) for ankylosing spondylitis (AS) based on randomized controlled trials (RCTs). This article claims to include a total of 5 RCTs, including original references 11-15 [in ref. 1]. There are problems with some of these RCTs.

Reference 11 in the original article is entitled "The Influence of BaDuan Jin on Somatic Function and Mental Health of the Patients with Ankylosing Spondylitis." However, no relevant literature has been found according to this title. According to the year of publication, authors, journal name, page, and volume, another paper with a similar title was found, entitled "The Influence of BaDuan Jin Combined with Wulao Qisun Prescriptions on Somatic Function and Mental Health of the Patients with Ankylosing Spondylitis" [2]. On reading this paper, this study divided patients into a treatment group and an observation group. Both groups were given meloxicam tablets, the observation group additional accepted Eight Brocade exercise combined with administration of Wulao Qisun (a traditional Chinese medicine) at the same time. Therefore, this article evaluates the efficacy of Eight Brocade exercise combined with traditional Chinese medi- cine, not the efficacy of Eight Brocade exercise. It cannot evaluate the role of Eight Brocade exercise in patients with AS alone. In terms of efficacy evaluation, Bath Ankylosing Spondylitis Disease Activity Index (BASDAI), Bath Ankylosing Spondylitis Functional Index (BASFI), visual analog scale, morning stiffness time, erythrocyte sedimentation rate (ESR), C-reactive protein (CRP), and symptom checklist (SCL-90) scores were evaluated in original reference 11 , but without any data on bone mineral density. An interesting result showed in the original article that "Only one study [ref. 11] suggests that Eight Brocade exercise plus drug therapy exerted a positive effect on bone mineral density compared with drug therapy alone" [1]. This result is groundless. The original text of reference 11 accompanied by an English abstract can be found in online supplementary material 1 (see www. karger.com/doi/10.1159/000510240 for all online suppl. material.

The accurate translation title of original reference 12 should be "Effect of Baduan Jin on inflammatory indexes of ankylosing spondylitis" [3], but this is not a big problem. The observation indicators in this study included ESR and CRP only [3]. It is questionable whether the authors can explain the source of BASFI, BASDAI, and the response rate shown in original Table 1 and the related Results section [1]. The original text of reference 12 accompanied by an English abstract can be found in online supplementary material 2.

The situation of original reference 13 is similar to that of original reference 11. By checking the authors, journal name, year of publication, volume, and other informa- 
tion, we are sure that the real title of the article should be "Clinical study of Baduan Jin combined with Bushen Qiangdu Juanbi decoction [a Chinese traditional medicine] in the treatment of Ankylosing Spondylitis," not "The efficacy of Baduanjin exercise for treating ankylosing spondylitis patients" in the original article [4]. The observation group in this study was given meloxicam tablets and the treatment group additional accepted Eight Brocade exercise combined with administration of Bushen Qiangdu Juanbi decoction. This study also cannot evaluate the role of Eight Brocade exercise alone in patients with AS. Here, a similar situation in original references 11 and 13 may partly or certainly explain the inconsistency in the title of the original text. Besides, neither BASFI nor BASDAI was actually reported in reference 13 , so it is confusing that this result is truly shown in the original article. The original text of reference 13 can be found in online supplementary material 3. Unfortunately, there is no English abstract for this article.

Similar to reference 12 , the accurate translation of reference 14 should be "The value of Baduan Jin in improving somatic function in patients with ankylosing spondylitis," but not "Baduanjin Qigong can improve body functions of patients with ankylosing spondylitis"; however, this is not a big problem [5]. The observation indicators of this study included physical function, pain index, thoracic mobility, Schober, finger to floor distance, occiput to wall distance, spinal stiffness, the overall evaluation of patients, the number of peripheral joints swelling and pain, and ESR. Indeed, BASFI, BASDAI, and CRP were not reported in this study. Therefore, where did the data of these indicators come from shown in the original article? The original text of reference 14 accompanied by an English abstract can be found in online supplementary material 4.

Besides, the studies from references 11-14 are hard to consider as RCTs. None of them are eligible for the CONSORT statement, which is a standard checklist when reporting RCTs [6]. In fact, they also do not claim to be RCTs themselves. The included studies in the original article for a non-English publication affect the evaluation of the quality of this article during the process of peer review.

Given the above, I consider the results of this article should be interpreted cautiously. Eight Brocade exercise may be of benefit to patients with AS; however, the results of this article seem not well-founded enough to support this conclusion.

\section{Conflict of Interest Statement}

The authors have no conflicts of interest to declare.

\section{Funding Sources}

None.

\section{Author Contributions}

Y.-H. He read the original article and wrote this comment.

\section{References}

1 Zou YY, Zhang HY, Xue L, Ye JJ, Hu GY. Traditional Chinese Eight Brocade Exercise Prescription for Ankylosing Spondylitis: A Quantitative Synthesis. Complement Med Res. 2020 May:1-5.

2 Fu JY, Tang R, Li XJ, Tian XM. The Influence of BaDuan Jin Combined with Wulao Qisun Prescriptions on Somatic Function and Mental Health of the Patients with Ankylosing Spondylitis. West J TCM. 2016;29(6):124-6.
3 Li YJ, Wei RB, Guo YR, Yin ZG. Effect of Baduan Jin on inflammatory indexes of ankylosing spondylitis. J North Pharm. 2012;9(6): $37-8$.

4 Liu J, Wei RB. Clinical study of Baduan jin combined with Bushen Qiangdu Juanbi decoction in the treatment of Ankylosing Spondylitis. J Changchun Univ TCM. 2012;28(6): 992-3.
5 Wei RB, Li YJ, Guo YR, Yin ZG. The value of Baduan Jin in improving somatic function in patients with ankylosing spondylitis. J North Pharm. 2012;9(6):67-8.

6 Schulz KF, Altman DG, Moher D; CONSORT Group. CONSORT 2010 statement: updated guidelines for reporting parallel group randomized trials. Ann Intern Med. 2010 Jun; 152(11):726-32. 